\title{
Design and Evaluation of a Mathematical Optimization Model for Traffic Signal Plan Transition Based on Social Cost Function
}

\author{
Rita Peñabaena-Niebles, ${ }^{1}$ Victor Cantillo, ${ }^{2}$ José Luis Moura, ${ }^{3}$ and Angel Ibeas ${ }^{3}$ \\ ${ }^{1}$ Department of Industrial Engineering, Universidad del Norte, Atlantico, Colombia \\ ${ }^{2}$ Department of Civil and Environmental Engineering, Universidad del Norte, Atlantico, Colombia \\ ${ }^{3}$ Department of Transport, Universidad de Cantabria, Cantabria, Spain \\ Correspondence should be addressed to Rita Peñabaena-Niebles; rpena@uninorte.edu.co
}

Received 20 January 2017; Revised 24 August 2017; Accepted 7 September 2017; Published 19 October 2017

Academic Editor: Jose E. Naranjo

Copyright (C) 2017 Rita Peñabaena-Niebles et al. This is an open access article distributed under the Creative Commons Attribution License, which permits unrestricted use, distribution, and reproduction in any medium, provided the original work is properly cited.

\begin{abstract}
Signal plan transition is the process of changing from one timing plan to another. It begins when the first intersection starts adjusting signal timing plans and ends when the last intersection completes adjusting signal timing plans. The transition between signal timing plans is required because traffic patterns change during the day. Therefore, it is necessary to modify signal timing parameters offset, phase split, and cycle length for different expectations of traffic volume. This paper presents an alternative and new mathematical model to enhance the performance of traffic signals coordination at intersections during the transition phase. This model is oriented to describe the transition regarding coordination parameters in all intersections of an arterial road for minimizing the social cost during the transition phase expressed in function of costs due to delays, fuel consumption, and air emissions. An ant colony algorithm was designed, coded, and simulated to find the optimal transition parameters using available data. The model was evaluated based on its ability to minimize social costs during the transition period. Results showed that the proposed method performs better than traditional ones.
\end{abstract}

\section{Introduction}

The rapid increase in urban traffic demand because of a growing population and economic development has led to an increase in the effects of traffic congestion and the associated externalities. A recent study revealed that the total traffic congestion cost in American urban areas would grow from \$101 billion dollars to \$133 billion dollars in 2015 to $\$ 199$ billion dollars in 2020. Delay times will grow to 6.1 billion hours in 2015 and 8.4 billion hours in 2020. The amount of fuel wasted due to congestion will increase to 2.5 billion gallons in 2015 and 4.5 billion gallons in 2020 [1]. Further, the 29 percent of total US greenhouse gas (GHG) emissions and over 5 percent of GHG emissions in the world are generated by the transportation sector [2]. The emissions of $\mathrm{CO}_{2}$ from fossil fuel combustion increased by 17 percent from 1990 to 2011. This led to an increase in overall emissions from transportation activities of 18 percent [3].
The statistics above show that, despite the efforts performed, there is still work to do in order to alleviate congestion problem and its implications. Efficient transportation system strategies have become a tool for mitigating traffic problems and improving mobility in cities. In addition, they may lead to a potential reduction in fuel consumption, GHG emissions, and delay. Improving traffic signals is one of the most valuable tools for decreasing total congestion and its effects. A study of the status of signals in USA indicated that basic signal improvements could achieve a 15 to 20 percent reduction in delay times, while advanced improvements can reduce them by up to 40 percent [4].

Many of researches [5-11] are directed towards increasing efficiency of traffic systems related to traffic signals. Improving traffic signals is one of the most effective tools for decreasing total congestion, travel time, delays, air pollution emissions, and fuel consumption. The coordination of traffic signals and optimization of timing plans and transition 
phases are often used to improve signals and traffic flow in arterial streets.

The signal timing is modified to achieve the coordination during the transition or process of changing from one signal timing plan to another new plan. Adjusting a timing plan may have an adverse impact on the level of service because traffic signals are operating with suboptimal values [12]. This increases travel time, delays, speed, stops, and greatest percentage of pollutant emissions and fuel consumption [13, 14], while minimizing the benefits that the new plan could achieve.

An optimal transition improves the efficiency of signal systems even in adaptive systems and preemption signal systems. In those systems, the need for better transition algorithms is heightened because the transition between timing plans occurs more frequently and its effects become more pronounced.

Two approaches have been identified in the literature to address the transition phase: applied methods, which are studies where the transition is attained by a sudden or gradual change of the parameters of the plan, while mathematical methods are based on optimizing operational measures of effectiveness during transition using mathematical models $[15,16]$. A recent study [15] concluded that most transition articles are focused on applied methods (78.3\%) and only a few addressed mathematical methods (21.7\%). Also, literature mainly focuses on investigating and analysing existing methods rather than presenting new solutions to optimize the transition phase.

Research projects conducted to date on transition strategies have centred on evaluating the impact and performance measures using different transition strategies on various traffic conditions. Most of the proposed transition methods were empirical methods based on experience, and they do not take advantage of current modelling, mathematical, and optimization techniques. During 1970 to 1981, most articles applied empirical methods, but only one mathematical method was developed [17]. In this work, the RAST algorithm was designed to minimize the duration of the transition period at critical nodes. Over the last two decades, the same trend remained. The amount of applied empirical methods was greater than the number of the developed mathematical methods. However; more attempts to design new mathematical methods were made: Abbas et al. [18] presented a traffic signal offset transitioning algorithm as an integrated optimization approach. Selekwa et al. [19] proposed a methodology to optimize traffic flow based on dynamic quadratic optimization. Lee and Williams [20] presented a nonlinear mathematical model that provides constrained delay minimization. Other studies about transition have oriented their efforts in optimizing time-of-day break points at a coordinated actuated traffic signal control system $[21,22]$

Existing mathematical methods aim to optimize only one measurement of efficiency and only minimize secondary factors such as parameter variation or time spent in transition. This can be seen as a disadvantage because complex methods are required to resemble prevailing conditions. This issue was pointed out by Selekwa et al. [19] and Penabaena-Niebles et al. [15] which stated that existing methods usually are not based on any optimization procedure and measurements of traffic flow are not optimized. As a result, the need of a more complex mathematical method able to resemble in a more accurate way the existing conditions arises. Consequently, there is an opportunity to design new mathematical models that provide solutions to the transition problem by simultaneously optimizing more than one measurement of efficiency and using advanced solution techniques.

The design of a mathematical model is presented in this paper in order to enhance the performance of traffic signals coordination at intersections during the transition phase. This model describes the transition in terms of coordination parameters of all intersections in an arterial road to minimize the social cost during the transition phase expressed in function of the delay cost, fuel consumption cost, and air emission cost. An ant colony algorithm determines the optimal parameters of coordination and the social cost due to transition, including user and environment costs. This method is considered because its main advantage is to allow the construction of the solution space, avoiding the generation of infeasible solutions. This issue is typical of evolutionary algorithms such as GA in the stage of crossover [20]. Also, its speed in processing time and suitability are considered relevant for dynamic problems [21-23] because of the complex characteristics of the problem under study and the importance of obtaining small response times.

The proposed model was designed to quantify the economic impact of a transition that benefits all modes of travel, improving not only measurements of traffic flow but also influencing the reduction of gas emissions and fuel consumption. Frequently, when a solution to the congestion problem is given, solutions are chosen separately, with the risk of causing the exacerbation of other problems. Only by considering multiple objectives, the full value of a mathematical model can be correctly addressed. The proposed model aims to generate a broad range of direct and indirect benefits. Primarily, it is intended to minimize the cost related to delay time, fuel, and gas emissions. However, this approach can also lead to a reduction of mileage that decreases vehicle depreciation, wear-and-tear, oil cost, and maintenance cost (repair and replacement).

The aim of this paper is to present a new method to address a complex problem, as is the transition between signal timing plans, and assess the impact regarding social cost and externalities generated during this phase, in relation to traditional methods, such as immediate, two cycles, and three-cycle transition.

\section{The Mathematical Model of Transition between Signal Timing Plans}

The mathematical model for transition is formulated as a nonlinear mathematical model where the transition is represented as a dynamic process in the time, oriented to maintain a progression and to smooth the adjusting the coordination parameters, offset, split, and cycle length of all intersections of an arterial. This model is limited to develop only the issues associated with the transition between signal 
timing plans and to measure the effectiveness of the proposed model in function of the social cost related to delay, fuel consumption, and gas emissions. This section presents the mathematical formulation of transition problem.

2.1. Model Approach and Basic Considerations. The proposed transition mathematical model has basic considerations. The model is oriented at minimizing delay, fuel consumption, and air emission gas during the transition period in function of the social cost established for each component of the model.

The objective function considers the effect of signals on delay, fuel consumption, and gas emissions at intersections. This assumption is important because the delay caused by a full stop may affect in a particular manner the vehicle acceleration and deceleration trajectories, fuel consumption, and air emissions. The presence of traffic signals causes interruptions in traffic flow because vehicles decelerate when they approach a stop line and signal turns red. Then, they stand still until the signal turns green. Finally, vehicles accelerate to pass the intersection [23]. This concept is used assuming no initial queue and no queue move up delay.

The constraint search spaced for transition procedure is defined by the strategies implemented by Lee and Williams [20]. Stepwise changes in offsets are associated with specific stepwise changes in background cycle length and vice versa during the transition phase. Thus, a common cycle length is maintained for the operations of a coordinated arterial, preserving the correlation between system cycle length and offset during the transition period. The authors demonstrated that this technique results in a more efficient transition. Precedence constraints during the transition period are developed to ensure that the total variations of control variables are within the levels of change established during the transition period. Finally, conditions for the standard NEMA dual ring structure for signal timings are contemplated.

\subsection{Transition Problem Variables and Objective Function.}

The proposed mathematical transition model has four (4) decision variables: the number of steps of the transition $n$, the offset shift $(\Delta \varphi)_{i j}$ in the intersection $i$ on each transition step $j$, the common cycle length shift $(\Delta C)_{j}^{c}$ on each transition step $j$, and the green effective timing $(\Delta g)_{i j m}$ in the intersection $i$ on each step $j$ for the movement $m$. The number of transition steps $n$ is the number of cycles needed to complete the transition. The offset change $(\Delta \varphi)_{i j}$ is the offset variation between transition steps, where offset is defined as the start of the green time on the coordinated phase of an intersection relative to the start of green time at a reference intersection. The common cycle length change $(\Delta C)_{j}^{c}$ is the common cycle length variation between steps during the transition, where the cycle length is the time needed to complete a rotation through all phases. It must be the same for all intersections in the coordination plan to maintain a consistent time based relationship [24], perform the effect of the transition, and ensure progression in the network. The split change $(\Delta g)_{i j m}$ is the variation of the green time in a phase within cycle between transitions steps.

The objective of the mathematical model in this research aims is to minimize the social cost during the transition period using the social cost function proposed by PeñabaenaNiebles et al. [25]. This model considers a generalized cost involving the effect of signals on the delay time, fuel consumption, and gas emissions at intersections. Therefore, the social cost function, which is applicable to any network of intersections with unsaturated traffic flow, is as presented in

$$
\begin{aligned}
\min \mathrm{SC} & =\sum_{j}^{n} \sum_{i}^{I} \mathrm{VT} \cdot \sum_{m}^{M}\left[\sum_{v}^{V} \mathrm{OR}_{v} \cdot D_{i j m v}\left(g_{i j m}, C_{i j}, q_{i j m v}\right)\right] \\
& +\sum_{j}^{n} \sum_{i}^{I} \sum_{m}^{M}\left[\sum_{v}^{V} \mathrm{OC}_{v} \cdot D_{i j m v}\left(g_{i j m}, C_{i j}, q_{i j m v}\right)\right]+\sum_{j}^{n} \sum_{i}^{I} \sum_{e}^{E}\left[\mathrm{ECG}_{e} \cdot \sum_{m}^{M} \sum_{v}^{V} \mathrm{GE}_{i j m v e}\left(g_{i j m}, C_{i j}, q_{i j m v}\right)\right],
\end{aligned}
$$

where $i$ is the intersection, $j$ is the transition step, $m$ are the movements by the lane or lanes, $v$ is the type of vehicle, and $e$ is the type of gas emission. In the model, the social cost (SC) is computed as the sum of driver or user cost and environment cost. In turn, the driver costs are given as an average delay by vehicle $\left(D_{i j m v}\right)$ and the fuel consumption $\left(\mathrm{FC}_{i j m v}\right)$ including the time cost per person in the vehicles (VT) and the vehicle operating costs $\left(\mathrm{OC}_{v}\right)$. The cost is estimated using the vehicle occupation time $\left(\mathrm{OR}_{v}\right)$, the average income, and a time value factor that turns average income into a value of time (VT) [26]. Meanwhile, the operating cost factor of the vehicles $\mathrm{OC}_{v}$ is formed by the fuel costs, tires, oil, repair, and maintenance. Similarly, the environment cost is the price or the monetary valuation of the greenhouse gas emissions $\left(\mathrm{ECG}_{e}\right)$ considering the global warming potential of the emission according to the type of gas to be evaluated $\left(\mathrm{GE}_{i j m v e}\right)$.

In this formulation, the Highway Capacity Manual (HCM) [27] delay model was selected to estimate average control delay per vehicle for each intersection in a corridor or arterial road, assuming no initial queue. This delay model is expressed in terms of cycle length, green time, progression factors, and traffic flow. In order to determine the delay in (1), the effect of the quality of progression in the coordinated movement was considered by calculating a $\mathrm{PF}_{i j m}$ following previous work by Lee [28]. Based on this, the percentage arrival under green is calculated assuming that vehicles released from upstream signals will reach the downstream 
signal in the free flow travel time, allowing a platoon of vehicles to move through several signals without stopping. The fuel consumption and gas emission components included are estimated according to the models proposed by Akçelik [29] and Frey et al. [30]. In order to evaluate the impact of signalized intersections on fuel consumption and air emissions, both components are based on the assumption of three independent amounts corresponding to three driving modes: idling, acceleration, and deceleration.

2.3. Feasibility Constraints. This section explains the conditions that define the space of possible solutions or restrictions in the proposed transition model. First, (2) sets out an equal cycle length for all intersections of the coordinated arterial defined as the common cycle length $\left(C_{j}^{c}\right)$ on each transition step $j$.

$$
C_{j}^{c}=C_{j-1}^{c}+(\Delta C)_{j}^{c} \quad \text { for } 1 \leq j \leq n
$$

Besides, (3)-(5) determine the signal timing transition plan or the values of parameters of coordination: cycle length $\left(C_{i j}\right)$ and phase green time $\left(g_{i j m}\right)$ for the movement $m$ and offset $\left(\varphi_{i j}\right)$ in function the change $(\Delta)$ for all intersections $i$ in each transition step $j$.

$$
\begin{gathered}
C_{i j}=C_{j}^{c}+(\Delta \varphi)_{i j} \quad \text { for } 1 \leq i \leq I, 1 \leq j \leq n \\
\varphi_{i j}=\varphi_{i j-1}+(\Delta \varphi)_{i j} \quad \text { for } 1 \leq i \leq I, 1 \leq j \leq n \\
g_{i j m}=g_{i j-1 m}+(\Delta g)_{i j m}
\end{gathered}
$$$$
\text { for } 1 \leq i \leq I, 1 \leq j \leq n, 1 \leq m \leq M
$$

Explicitly, (2) and (3) allow the existence of system or common cycle lengths $\left(C_{j}^{c}\right)$ and consider the relationship between offset $\left(\varphi_{i j}\right)$ and cycle length $\left(C_{i j}\right)$, in all intersections $i$ in each transition step $j$. This is necessary to provide progressive operation between intersections.

Equations (6) ensure that the total variation in the parameters coordination is inside of the level of change established for old and new signal plan (before and after transition signal plan). For this, it is necessary that the optimal signal timing plan parameters are determined before and after the transition period.

$$
\begin{aligned}
& \sum_{j=1}^{n}(\Delta C)_{j}^{c}=C_{i f}-C_{i o} \quad \text { for } 1 \leq i \leq I, 1 \leq j \leq n \\
& \sum_{j=1}^{n}(\Delta \varphi)_{i j}=\varphi_{i f}-\varphi_{i o} \quad \text { for } 1 \leq i \leq I, \quad 1 \leq j \leq n \\
& \sum_{j=1}^{n}(\Delta g)_{i j m}=g_{i f m}-g_{i o m}
\end{aligned}
$$

$$
\text { for } 1 \leq i \leq I, 1 \leq j \leq n, 1 \leq m \leq M \text {. }
$$

In turn, (7) are precedence constraints and allow that coordination parameters are within the interval between previous step cycle length and cycle length after the transition.

$$
\begin{gathered}
C_{i j-1} \leq C_{i j} \leq C_{i f} \quad \text { for } 1 \leq i \leq I, 1 \leq j \leq n \\
\varphi_{i j-1} \leq \varphi_{i j} \leq \varphi_{i f} \quad \text { for } 1 \leq i \leq I, 1 \leq j \leq n \\
g_{i j-1 m} \leq g_{i j m} \leq g_{i f m} \quad \text { for } 1 \leq i \leq I, 1 \leq j \leq n .
\end{gathered}
$$

The set of constrains defined by (8) are the conditions for the standard NEMA dual ring structure for signal timings, where $y_{i j m}$ and $r_{i j m}$ are the yellow and red time of intersection $i$ at the transition step $j$ for movement $m$, respectively.

$$
C_{i j}=\sum_{m=1}^{4}\left(g_{i j m}+y_{i j m}+r_{i j m}\right)
$$

$$
\text { for } 1 \leq i \leq I, 1 \leq j \leq n
$$

$$
\begin{array}{ll}
g_{i j 1}+g_{i j 2}=g_{i j 5}+g_{i j 6} & \text { for } 1 \leq i \leq I, 1 \leq j \leq n \\
g_{i j 3}+g_{i j 4}=g_{i j 7}+g_{i j 8} & \text { for } 1 \leq i \leq I, 1 \leq j \leq n .
\end{array}
$$

Finally, (9) sets the maximum number of transition steps regarding the analysed period $(T f)$ and the initial cycle length $C_{i 0}$.

$$
1 \leq n \leq \frac{T f}{C_{i 0}} \quad \text { for } 1 \leq i \leq I
$$

\section{Ant Colony Optimization}

For solving the mathematical model for the transition between signal timing plans, the problem will be represented as an Ant Colony Optimization (ACO) problem, where the ACO algorithm is designed to find the transition parameters that minimize social cost.

ACO is inspired by the cooperative behaviour of real ant colonies and their process to find a food source [31]. These ants deposit a chemical substance, called pheromone, which is accumulative and evaporative, in order to mark some favourable paths that other members of the colony should follow. ACO is an iterative algorithm, in which each iteration, a set of possible solutions, is constructed using artificial ants [32]. At each step, the ants travel the shorter path following the pheromone trail left by previous ants. The pheromone trails tend to evaporate, which means that it decreases when few ants travel on the path and increases when many ants travel on it. Consequently, the pheromone evaporates faster on long paths and short paths occur opposite. This phenomenon causes that all the ants tend to travel the shortest path.

Ant colony optimization was designed by Dorigo and Caro [33] as a metaheuristic or an algorithm that can be applied to different optimization problems with some modifications. Initially, this algorithm was used to solve single objective problems based on the traveling salesman problem (TSP). However, recently it has been applied to combinatorial optimization problems such as distribution 
scheduling problem, vehicle routing problem, and railroad blocking problem, among other applications [34-38]. ACO has been applied to transportation systems optimization, but these works have been oriented to find optimal signal setting in function of traffic demand [39-41].

In this research, ACO generates the coordination parameters during transition cycles, which are a signal plan for each step to achieve the transition. Then, the social cost is determined using the transition plan to evaluate the actual transition plan and improving the set of parameters coordination in the next iteration. This is repeated until the solution converges to a minimum value or a previously defined number of iterations are reached. The next subsections describe in detail the implementation of ACO algorithm for solving the minimization problem.

3.1. Ant Colony Preparation. The algorithm begins assuming a number of ants in the colony $(C)$. In this problem, three subtypes of ants (AntD, AntF, and AntE) are defined and all of them are associated with delay cost, fuel consumption cost, and air emission cost, respectively. The number of ants for each subtype is the third part of the total population or ants in the colony; that is, all components of the objective function are equally important.

3.2. Constructing Ant Solution. Ants are randomly placed on a solution space that represents a multidimensional structure where all possible solutions of the problem are defined. A set of possible discrete values for each of the problem variables is assumed. For this, it is necessary to set a single solution. In this case, the proposed mathematical transition model has four (4) decision variables: number of transition steps $n$, offset change $(\Delta \varphi)_{i j}$, common cycle length change $(\Delta C)_{j}^{c}$, and effective green time or split change $(\Delta g)_{i j m}$. Cycle length $\left(C_{i j}\right)$ can be calculated from $(\Delta C)_{j}^{c}$ and offset change $(\Delta \varphi)_{i j}$ using (3). Concurrently, split change $(\Delta g)_{i j m}$ can be obtained from cycle length $\left(C_{i j}\right)$ using (5) and (8). Steps $n$, common cycle length change $(\Delta C)_{j}^{c}$, and offset change $(\Delta \varphi)_{i j}$ must be known to find a solution. This means that a set of different values for each of the variables should be obtained. The decision variable $n$ is restricted as an integer number (see (9)) and the common cycle length change $(\Delta C)_{j}^{c}$ and offset change $(\Delta \varphi)_{i j}$ are two multidimensional arrays. Therefore, there are much more possible ways of being generated.

Nevertheless, for indices $i$ and $j$, the values of $(\Delta C)_{j}^{c}$ and $(\Delta \varphi)_{i j}$ are restricted by (2) and (4). Therefore, they can only vary independently through index $j$. To avoid generating random values on each one of the $j$ submatrices of $(\Delta C)_{j}^{c}$ and $(\Delta \varphi)_{i j}$, these values are interpolated with an exponential function. At the same time, this allows that the obtained solutions are analysed more properly in order to infer rules that lead to a better understanding of them.

Considering the above assumptions, (2) and (4) are redefined as follows:

$$
C_{j}^{c}=C_{i o}+\left(C_{i f}-C_{i o}\right)\left(\frac{j}{n}\right)^{c \text { pow }}
$$

for $1 \leq i \leq I, 1 \leq j \leq n$

$$
\begin{aligned}
& \varphi_{i j}=\varphi_{i o}+\left(\varphi_{i f}-\varphi_{i o}\right)\left(\frac{j}{n}\right)^{\varphi \text { pow }} \\
& \qquad \text { for } 1 \leq i \leq I, 1 \leq j \leq n
\end{aligned}
$$

The values of $C_{i o}$ and $C_{i f}$ are given as parameters for all interceptions $i \in I$, as well as all the values for $\varphi_{i o}$ and $\varphi_{i f}$ for all interceptions $i \in I$. Consequently, the only unknown variables in equations are cpow and $\varphi$ pow, which are exponents that allow the output of the function to vary according to them. Therefore, a solution can be generated just with three parameters: $n, c$ pow, and $\varphi$ pow, which are the three dimensions in the space solution.

Different values of cpow and $\varphi$ pow yield different interpolations for a solution. One of the properties of the exponential functions is as follows:

$$
x^{a} \leq 1 \quad \text { if } a \geq 0 \forall x \in[0,1] .
$$

If $g$ pow $\geq 0 \varphi$ pow $\geq 0$, then for $1<j<n$

$$
\begin{aligned}
&(\Delta \varphi)_{i 1} \leq(\Delta \varphi)_{i j} \leq(\Delta \varphi)_{i n}, \\
&(\Delta C)_{i 1} \leq(\Delta C)_{j}^{c} \leq(\Delta C)_{i n} .
\end{aligned}
$$

Also, due to the fact that the exponential function is monotonically increasing in its entire domain, (2) and (4) are always respected.

Then, the solution space is a three-dimensional structure that contains all possible triplets composed by $n, c$ pow, and $\varphi$ pow; thus

$$
\begin{aligned}
S= & \{(n, c, \varphi) ; n \in N, \text { cpow } \in \text { CPow, } \varphi \text { pow } \\
& \in \Phi \text { Pow }\},
\end{aligned}
$$

where $N$ is the set of all the possible values for $n$ and CPow and ФPow are all the possible values for $c$ pow and $\varphi$ pow, respectively.

The values of cpow and $\varphi$ pow affect the interpolation in (10). For values below one, they define an initially fast growing and lastly slow growing curve, and values above one describe the opposite. Finally, when cpow or $\varphi$ pow are equal to 1 , they yield linear interpolation functions.

Hence a reasonable range for $\Phi$ Pow and $C P$ Pow, which allows the comparison of different behaviours of the objective function, is

$$
\{x, 1 \geq x \geq 10, x \in \mathbb{N}\} \cup\left\{\frac{1}{x}, 1>x \geq 10, x \in \mathbb{N}\right\} .
$$

However, these values can vary because they are parameters in the constructor of solution space.

3.3. Defining Probabilities. Once the ant colony and the solution space are instantiated, ants can start to move. Ants of different types are randomly distributed through the solution space at the beginning of the algorithm. Each ant must be initialized with values for its coordinates in $n$, cpow, and $\varphi$ pow axes. They are used as parameters to build the solution corresponding to that location in the space. 


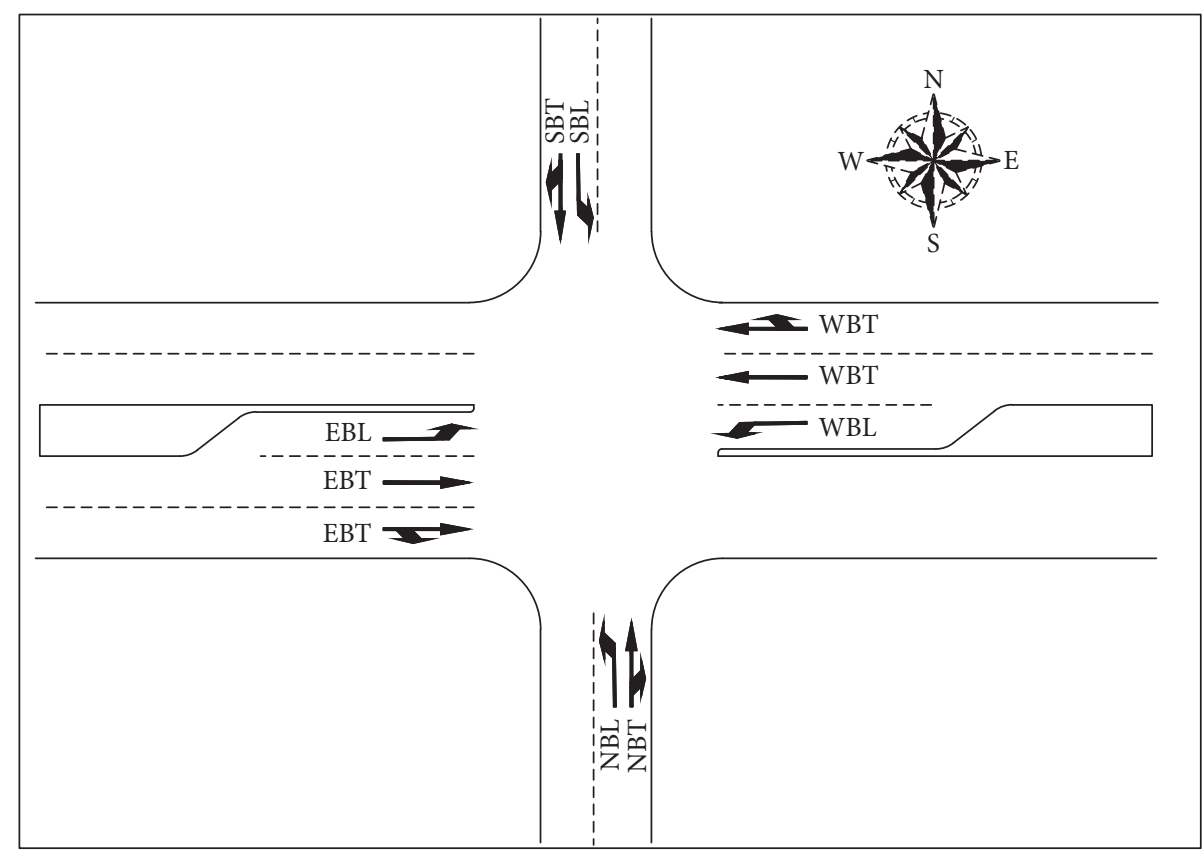

FIGURE 1: Layout and movement of the signalized intersections.

A move of an ant means a change in its position from one point in the solution space to another, generating a new solution. However, there are some restrictions: first, the same ant must not have visited the same position before and second, that point must be in the neighbour. These points are obtained either by adding or by subtracting one unit to exclusively one of the coordinates $n$, cpow, and gpow of the current position. The number of movements for any ant is given as a parameter of the algorithm and referred as iterations.

In ACO algorithm, the probability that an ant moves to more than one neighbour point is given by the following expression:

$$
p_{(n, c \text { pow }, \varphi \text { pow })}^{k}=\frac{\left(\tau_{n, \text { cpow }, \varphi \text { pow }}^{\alpha}\right)\left(\eta_{n, c \text { pow }, \varphi \text { pow }}^{\beta}\right)}{\sum\left(\tau_{n, c \text { pow }, \varphi \text { pow }}^{\alpha}\right)\left(\eta_{n, \text { cpow }, \varphi \text { pow }}^{\beta}\right)},
$$

where $\tau_{n, c \text { pow, } \varphi \text { pow }}$ is the amount of pheromone deposited in the point ( $n$, cpow, $\varphi$ pow) of the solution space, $\alpha \geq 0$ is a parameter that controls the influence of $\tau_{n, \text { cpow }, \varphi \text { pow }}, \eta_{n, \text { cpow }, \varphi \text { pow }}$ is the attractiveness of a point in the space, and $\beta \geq 1$ is a parameter to control the influence of $\eta_{n, \text { cpow, } \varphi \text { pow }}$.

3.4. Selection and Evaluation of Paths. The class solution provides all the functionality needed to get the output value of the social cost. Ants define this value, depending on their type (AntD, AntF, and AntE) to obtain the attractiveness of their neighbour points in the solution space as expressed in (16), in which $f_{o}$ is the objective function that corresponds to the ant type

$$
\eta_{n, c \text { pow }, \varphi \text { pow }}=f_{o}(n, c \text { pow }, \varphi \text { pow }) .
$$

3.5. Updating Pheromone. Each time that ants move through the solution space, they leave a trail of pheromone. It is accumulated in a three-dimensional floating point array within the class solution space. This matrix is updated as follows. For each ant, a trail of pheromone is added to the same point of the solution space where the ant is located in the neighbour points:

$$
\tau_{n, \mathrm{cpow}, \varphi \mathrm{pow}}=\tau_{n, \mathrm{cpow}, \varphi \mathrm{pow}}+\lambda * \delta,
$$

where $\lambda$ represents the pheromone trail left by an ant and $\delta$ a value inversely proportional to the distance of the neighbour point to the original point where the ant was located.

At the end of the iteration, the trail evaporates based on (16), where $\rho$ is the pheromone evaporation coefficient.

$$
\tau_{n, c \text { pow }, \varphi \text { pow }}=(1-\rho) \tau_{n, \text { ppow }, \varphi \text { pow }} .
$$

The ACO process is finished either if the number the iterations is reached or until the process converges. This means that all the ants choose the same best path.

\section{Numerical Example}

A hypothetical linear network with three signalized intersections adapted from the case presented by Lee and Williams [20] was used to execute the proposed mathematical model for transition. Also, the effects of transition timing plans optimized in stops, delays, and its impact on the social cost during the transition period were evaluated.

As shown in Figure 1, each approach of the intersections is configured with a single exclusive left turn lane, 3 full lanes on the main street, and 1 full lane on side streets. The turning movement ratios for each intersection are set to $15 \%, 75 \%$, 
TABLE 1: Data before the transition in the numerical example.

\begin{tabular}{|c|c|c|c|c|c|c|c|c|c|c|c|}
\hline \multicolumn{12}{|c|}{ Signal timing before transition } \\
\hline \multirow{2}{*}{ Intersection } & Phase & 1 & 2 & 3 & 4 & 5 & 6 & 7 & 8 & \multirow{2}{*}{ Cycle length } & \multirow{2}{*}{ Offset } \\
\hline & Movement & WBL & EBT & NBL & SBT & EBL & WBT & SBL & NBT & & \\
\hline \multirow{2}{*}{1} & Optimized green phase (s) & 13 & 20 & 9 & 23 & 13 & 20 & 8 & 24 & 65 & 31 \\
\hline & Flow rate (veh/s) & 177 & 866 & 77 & 361 & 153 & 1002 & 63 & 440 & - & - \\
\hline \multirow{2}{*}{2} & Optimized green phase (s) & 11 & 22 & 9 & 23 & 13 & 20 & 9 & 23 & 65 & 0 \\
\hline & Flow rate (veh/s) & 151 & 1022 & 78 & 362 & 180 & 853 & 63 & 443 & - & - \\
\hline \multirow{2}{*}{3} & Optimized green phase (s) & 10 & 24 & 9 & 22 & 14 & 20 & 8 & 23 & 65 & 31 \\
\hline & Flow rate (veh/s) & 132 & 1225 & 82 & 379 & 216 & 743 & 67 & 464 & - & - \\
\hline
\end{tabular}

TABLE 2: Data after the transition in the numerical example.

\begin{tabular}{|c|c|c|c|c|c|c|c|c|c|c|c|}
\hline \multicolumn{12}{|c|}{ Signal timing after transition } \\
\hline \multirow{2}{*}{ Intersection } & Phase & 1 & 2 & 3 & 4 & 5 & 6 & 7 & 8 & Cycle length & Offset \\
\hline & Movement & WBL & EBT & NBL & SBT & EBL & WBT & SBL & NBT & & \\
\hline \multirow{2}{*}{1} & Optimized green phase (s) & 29 & 31 & 11 & 44 & 17 & 43 & 13 & 42 & 115 & 69 \\
\hline & Flow rate (veh/s) & 294 & 1014 & 91 & 633 & 178 & 1671 & 111 & 517 & - & - \\
\hline \multirow{2}{*}{2} & Optimized green phase (s) & 23 & 35 & 12 & 45 & 20 & 38 & 13 & 44 & 115 & 0 \\
\hline & Flow rate (veh/s) & 246 & 1165 & 87 & 603 & 205 & 1394 & 107 & 493 & - & - \\
\hline \multirow{2}{*}{3} & Optimized green phase (s) & 21 & 38 & 11 & 45 & 22 & 36 & 12 & 45 & 115 & 69 \\
\hline & Flow rate (veh/s) & 208 & 1367 & 86 & 601 & 241 & 1180 & 106 & 491 & - & - \\
\hline
\end{tabular}

TABLE 3: Fuel consumption and gas emission rates according to the driving mode and vehicle type.

\begin{tabular}{cccccccccc}
\hline & & Fuel $(\mathrm{g} / \mathrm{sec})$ & $\mathrm{CO}(\mathrm{g} / \mathrm{sec})$ & $\mathrm{GPW}_{\mathrm{CO}}$ & $\mathrm{HC}(\mathrm{g} / \mathrm{sec})$ & $\mathrm{GPW}_{\mathrm{HC}}$ & Nox $(\mathrm{g} / \mathrm{sec})$ & $\mathrm{GPW}_{\mathrm{NO} x}$ & $\mathrm{O} . \mathrm{rate}$ \\
\hline \multirow{3}{*}{ Light vehicle } & Acceleration & 0.5028 & 0.4972 & 3 & 0.6321 & 12 & 0.5847 & 40 \\
& Deceleration & 0.4342 & 0.1365 & 3 & 0.2488 & 12 & 0.4127 & 40 & 1.3 \\
& Idling & 0.0714 & 0.3720 & 3 & 0.2343 & 12 & 0.0991 & 40 & \\
\hline \multirow{3}{*}{ Heavy vehicle } & Acceleration & 1.33 & 0.6969 & 3 & 0.6383 & 12 & 0.3100 & 40 \\
& Deceleration & 1.17 & 0.6534 & 3 & 0.6941 & 12 & 0.2628 & 40 & 1.2 \\
& Idling & 0.259 & 0.7065 & 3 & 0.2279 & 12 & 0.2326 & 40 & \\
\hline
\end{tabular}

and $10 \%$ for the left turn, through, and right turn movements, respectively.

Tables 1 and 2 show traffic flow and optimized signal timing before and after the transition, respectively.

The parameters to determine the social cost of the model proposed are used from the case study presented by Peñabaena-Niebles et al. [25, 42]. The time cost per person (VT) is $\$ 9.432 /$ hour. The vehicle operating cost per gallon of fuel is $\$ 7.25 /$ gal, and the marginal social cost of greenhouse gas emissions for carbon dioxide $\left(\mathrm{CO}_{2}\right)$ is $\$ 25 / \mathrm{tC}$ (which is taken from a study performed by Fankhauser [43]). The global warming potential (GWP) for $\mathrm{CO}, \mathrm{HC}$, and $\mathrm{NO}_{x}$, the weighted average of carbon monoxide (CO), hydrocarbon $(\mathrm{HC})$, and nitrogen oxide $\left(\mathrm{NO}_{x}\right)$ rates and fuel consumption rates in each driving and the occupation rates for each type of vehicle are shown in Table 3.

The ACO procedure described previously was applied to find the optimal transition. The implementation was made coding the algorithm using Python 2.7. After setting up the objective function and the ACO algorithm, two (2) tests were made to measure the correspondence between algorithm parameters and objective function key values.
First, the algorithm was applied to determine the impact of the number of ants used in the values of the social cost. For this test, the number of ants was ranged between 1 and 100 and the number of iterations was fixed at 30 . The test was run 10 times and the average of the obtained results was calculated (Figure 2(a)). Subsequently, the impact of number of iterations in the values of objective function was measured. For this, the number of iterations was ranged between 1 and 100 and the number of ants was fixed at 25 . The tests were run 10 times and the results are detailed in Figure 2(b). For both tests, parameters are set as follows: $\lambda=0.85, \delta=0.2$, and $\rho=0.04$. As Figure 2 shows, the model requires for convergence about 30 ants and 45 iterations.

From Figure 2, it was concluded that raising both parameters, ants or iterations, would cause a lower value of the average cost social and delay. However, both results are more sensitive to the number of iterations. This is because the iteration count decided how much coverage each ant will have, thus allowing them to achieve more optimal solutions. The number of ants affects how much areas of the solution space will be covered at the start of the algorithm. Thus, an appropriate set of parameters to the ACO algorithm was 
TABLE 4: Transition timing plan with ACO procedure.

\begin{tabular}{|c|c|c|c|c|c|c|c|c|c|c|c|}
\hline \multicolumn{12}{|c|}{ Green movement } \\
\hline \multirow[t]{2}{*}{ Step } & \multirow[t]{2}{*}{ Intersection } & 1 & 2 & 3 & 4 & 5 & 6 & 7 & 8 & Cycle length & Offset \\
\hline & & WBL & EBT & NBL & SBT & EBL & WBT & SBL & NBT & & \\
\hline \multirow{3}{*}{ Before } & 1 & 13 & 20 & 9 & 23 & 13 & 20 & 8 & 24 & 65 & 31 \\
\hline & 2 & 11 & 22 & 9 & 23 & 13 & 20 & 9 & 23 & 65 & 0 \\
\hline & 3 & 10 & 24 & 9 & 22 & 14 & 20 & 8 & 23 & 65 & 31 \\
\hline \multirow{3}{*}{1} & 1 & 15 & 21 & 9 & 25 & 13 & 23 & 8 & 26 & 66 & 31 \\
\hline & 2 & 11 & 22 & 9 & 24 & 13 & 20 & 9 & 24 & 66 & 0 \\
\hline & 3 & 11 & 25 & 9 & 26 & 14 & 22 & 8 & 27 & 66 & 31 \\
\hline \multirow{3}{*}{2} & 1 & 15 & 22 & 9 & 27 & 14 & 23 & 8 & 27 & 69 & 32 \\
\hline & 2 & 11 & 22 & 9 & 27 & 13 & 20 & 9 & 27 & 68 & 0 \\
\hline & 3 & 13 & 25 & 9 & 27 & 14 & 24 & 9 & 27 & 69 & 32 \\
\hline \multirow{3}{*}{3} & 1 & 18 & 25 & 10 & 31 & 15 & 29 & 9 & 31 & 73 & 33 \\
\hline & 2 & 13 & 26 & 10 & 30 & 15 & 25 & 9 & 30 & 71 & 0 \\
\hline & 3 & 14 & 28 & 10 & 30 & 17 & 26 & 9 & 30 & 73 & 33 \\
\hline \multirow{3}{*}{4} & 1 & 18 & 25 & 10 & 33 & 15 & 29 & 9 & 33 & 77 & 34 \\
\hline & 2 & 15 & 26 & 10 & 30 & 16 & 25 & 10 & 30 & 74 & 0 \\
\hline & 3 & 16 & 28 & 11 & 32 & 18 & 27 & 10 & 32 & 77 & 34 \\
\hline \multirow{3}{*}{5} & 1 & 22 & 26 & 11 & 32 & 16 & 32 & 10 & 33 & 82 & 35 \\
\hline & 2 & 19 & 29 & 11 & 31 & 17 & 31 & 11 & 31 & 78 & 0 \\
\hline & 3 & 16 & 32 & 11 & 34 & 18 & 31 & 10 & 34 & 82 & 35 \\
\hline \multirow{3}{*}{6} & 1 & 23 & 27 & 11 & 35 & 16 & 35 & 11 & 34 & 89 & 37 \\
\hline & 2 & 19 & 31 & 11 & 34 & 18 & 32 & 11 & 34 & 83 & 0 \\
\hline & 3 & 17 & 33 & 11 & 37 & 19 & 30 & 11 & 38 & 89 & 37 \\
\hline \multirow{3}{*}{7} & 1 & 25 & 30 & 11 & 39 & 16 & 38 & 12 & 39 & 101 & 40 \\
\hline & 2 & 19 & 31 & 11 & 37 & 18 & 32 & 11 & 37 & 92 & 0 \\
\hline & 3 & 21 & 33 & 11 & 40 & 20 & 33 & 12 & 40 & 101 & 40 \\
\hline \multirow{3}{*}{8} & 1 & 26 & 30 & 11 & 42 & 17 & 40 & 12 & 40 & 115 & 44 \\
\hline & 2 & 19 & 31 & 11 & 37 & 18 & 32 & 11 & 37 & 102 & 0 \\
\hline & 3 & 21 & 35 & 11 & 42 & 20 & 34 & 12 & 43 & 115 & 44 \\
\hline \multirow{3}{*}{ After } & 1 & 29 & 31 & 11 & 44 & 17 & 43 & 13 & 42 & 115 & 69 \\
\hline & 2 & 23 & 35 & 12 & 45 & 20 & 38 & 13 & 44 & 115 & 0 \\
\hline & 3 & 21 & 38 & 11 & 45 & 22 & 36 & 12 & 45 & 115 & 69 \\
\hline
\end{tabular}



(a)

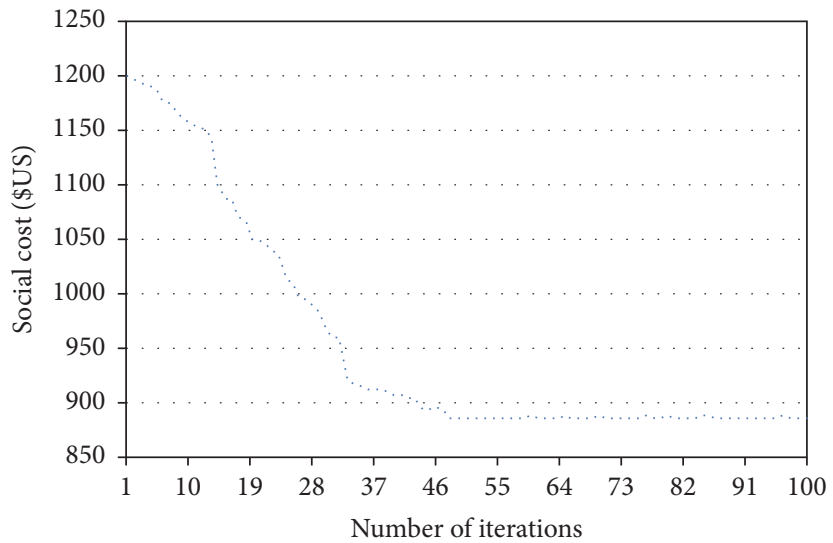

(b)

Figure 2: ACO converge using social cost. 
TABLE 5: Results of simulation for study system.

\begin{tabular}{|c|c|c|c|c|c|c|c|c|c|c|}
\hline & \multicolumn{2}{|c|}{ Social cost } & \multicolumn{2}{|c|}{ Time value cost } & \multicolumn{2}{|c|}{ Vehicle operating cost } & \multicolumn{2}{|c|}{ Gas emission cost } & \multicolumn{2}{|c|}{ Delay } \\
\hline & US \$ & $\%$ & US \$ & $\%$ & US \$ & $\%$ & US \$ & $\%$ & Sec/veh & $\%$ \\
\hline Proposed (ACO) & 879.0 & - & 778.2 & - & 91.3 & - & 9.5 & - & 25.82 & - \\
\hline Immediate & 1135.5 & $33 \%$ & 1006.6 & $23 \%$ & 117.1 & $22 \%$ & 11.9 & $20 \%$ & 27.19 & $5 \%$ \\
\hline Two & 1114.1 & $23 \%$ & 987.7 & $21 \%$ & 114.7 & $20 \%$ & 11.7 & $19 \%$ & 27.02 & $4 \%$ \\
\hline Three & 1141.2 & $21 \%$ & 1010.7 & $23 \%$ & 118.6 & $23 \%$ & 11.9 & $21 \%$ & 27.21 & $5 \%$ \\
\hline
\end{tabular}

TABLE 6: Results of simulation for gas emissions.

\begin{tabular}{|c|c|c|c|c|c|c|}
\hline & \multicolumn{2}{|c|}{$\mathrm{HC}$} & \multicolumn{2}{|c|}{ Nox } & \multicolumn{2}{|c|}{$\mathrm{CO}$} \\
\hline & g & $\%$ & g & $\%$ & g & $\%$ \\
\hline Proposed (ACO) & $28,192.8$ & - & $92,012.9$ & - & $6,453.5$ & - \\
\hline Immediate & $35,439.8$ & $20.4 \%$ & $114,472.9$ & $19.6 \%$ & $8,102.3$ & $20.3 \%$ \\
\hline Two & $35,038.4$ & $19.5 \%$ & $113,028.3$ & $18.6 \%$ & $7,973.7$ & $19.1 \%$ \\
\hline Three & $35,778.7$ & $21.4 \%$ & $115,232.7$ & $20.2 \%$ & $8,085.7$ & $20.2 \%$ \\
\hline
\end{tabular}

TABLE 7: Results of simulation for main streets.

\begin{tabular}{|c|c|c|c|c|c|c|c|c|c|c|}
\hline & \multicolumn{2}{|c|}{ Social cost } & \multicolumn{2}{|c|}{ Time value cost } & \multicolumn{2}{|c|}{ Vehicle operating cost } & \multicolumn{2}{|c|}{ Gas emission cost } & \multicolumn{2}{|c|}{ Delay } \\
\hline & US \$ & $\%$ & US \$ & $\%$ & US \$ & $\%$ & US \$ & $\%$ & Sec/veh & $\%$ \\
\hline Proposed (ACO) & 538.5 & - & 476.7 & - & 56.0 & - & 5.8 & - & 15.82 & - \\
\hline Immediate & 685.1 & $21 \%$ & 607.3 & $22 \%$ & 70.6 & $21 \%$ & 7.2 & $19 \%$ & 16.40 & $4 \%$ \\
\hline Two & 676.5 & $20 \%$ & 591.2 & $19 \%$ & 77.8 & $28 \%$ & 7.5 & $23 \%$ & 16.91 & $6 \%$ \\
\hline Three & 819.4 & $34 \%$ & 731.0 & $35 \%$ & 80.8 & $31 \%$ & 7.6 & $24 \%$ & 17.71 & $11 \%$ \\
\hline
\end{tabular}

TABLE 8: Results of simulation for secondary streets.

\begin{tabular}{lcccccccccc}
\hline & \multicolumn{2}{c}{ Social cost } & \multicolumn{2}{c}{ Time value cost } & \multicolumn{2}{c}{ Vehicle operating cost } & \multicolumn{2}{c}{ Gas emission cost } & \multicolumn{2}{c}{ Delay } \\
& US \$ & $\%$ & US \$ & $\%$ & US \$ & $\%$ & US \$ & $\%$ & Sec/veh & $\%$ \\
\hline Proposed (ACO) & 340.6 & - & 301.5 & - & 35.4 & - & 3.7 & - & 10.0 & - \\
Immediate & 450.3 & $24 \%$ & 399.2 & $24 \%$ & 46.4 & $24 \%$ & 4.7 & $22 \%$ & 10.8 & $7 \%$ \\
Two & 437.6 & $22 \%$ & 396.5 & $24 \%$ & 36.9 & $4 \%$ & 4.2 & $13 \%$ & 10.1 & $1 \%$ \\
Three & 321.8 & $-6 \%$ & 279.7 & $-8 \%$ & 37.8 & $6 \%$ & 4.3 & $15 \%$ & 9.5 & $-5 \%$ \\
\hline
\end{tabular}

defined as 25 ants and 60 iterations that allow finding the best social cost values associated with the transition.

Table 4 shows the transition timing plans following the ACO procedure under these conditions. The result obtained is a transition timing plan for nine periods.

To define the effectiveness and environmental impact of this new approach, a simulation was conducted using AIMSUN 8.1 to compare the proposed transition plan proposed (Tables 6-8) with traditional methods, such as immediate, two, and three cycles.

Table 5 shows the numerical comparisons of social cost between transition plans obtained with ACO, immediate, two, and three cycles methods. The results achieved in the simulation with transition plan obtained with ACO algorithm were \$US 879 for the total social cost, \$US 778.20 for time costs, \$US 91.3 for vehicle operating costs, and \$US 9.50 for the cost of $\mathrm{CO}, \mathrm{HC}$, and $\mathrm{NO}_{x}$ emissions.

Improvements around $20 \%$ in vehicle operating costs and gas emission costs indicate the good performance of the proposed method from an environmental point of view.
Although decreases in delay are around 5\%, it is important to stand out great improvements for the users regarding time cost of about $20 \%$. This occurs because the model proposed takes into account the occupancy rate or the number of users of the system.

In addition, it can be observed in Table 5 that the proposed method offers the best performance in delay times in comparison with the other studied methods. The average control delay per vehicle when the transition occurs in nine steps was 25.82 seconds/vehicle. The second better method was two-cycle.

Moreover, Table 6 shows the environmental impact of each transition method regarding the greenhouse gases and emissions to the atmosphere. As can be seen, the proposed method is the best for the environment followed by the twocycle method. In general, reductions of about $20 \%$ occur when the proposed method is used over the other methods.

To evaluate the performance of proposed method over the main and secondary streets, the system was divided into two subsystems. Tables 7 and 8 present the results 
of the simulation for the principal and secondary streets, respectively. Table 7 shows that the proposed method gives the best indicators of the components of the social cost function. The second best method for the transition is the method of the two cycles. The method of the three cycles is the only one with the worst indicators for the social cost function.

Table 8 concludes that, for secondary streets, the method of three cycles delivers the best results for the social cost, time value, and delays for this subsystem, above the proposed method. However, the environmental component obtained from the proposed method retains its superiority.

\section{Conclusions}

This study proposed a nonlinear mathematical model to improve the performance of the transition between signal timing plans at coordinated intersections using a multiobjective function that considers the social cost in terms of delays, fuel consumption, and air emissions. The model constitutes a new alternative for evaluating the social impact of transition in signal intersections as it considers the user and environmental costs as performance measures, allowing a more accurate estimate of externalities.

An ACO algorithm was proposed for designing transition timing plans that minimize the social cost. The objective of this procedure was to reduce social cost efficiently by appropriate changes of offset, cycle length, and green phase parameters in a complex mathematical problem of the transition.

The designed model can be included into traffic control systems for reducing the effects of congestion during the transition.

Finally, it can be stated that the proposed method showed better performance measures. It obtained improvements around $20 \%$ in the social cost and $5 \%$ in the delay. Also, the proposed method favours the social cost function for the complete system, giving the best performance for the principal streets and a good performance for the secondary ones. It also showed a good performance for the cases under study based on an environmental point of view.

Future studies can be focused on the validation of the proposed method under saturation conditions and in responsive traffic systems. On the other hand, the effects of the proposed method have to be evaluated using a great network to confirm its efficiency regarding the reduction of the social cost. Similarly, it is important to perform a study to compare processing times and performance in the social cost function using different new optimization techniques.

\section{Variables}

$i$ : The intersection

j: $\quad$ The transition step

$n$ : The total number of transition steps

$m$ : Phase or movements by lane groups

$v$ : Type of vehicle

$e: \quad$ Type of emission (gas)

VT: Value of time (\$US/hour)

$\mathrm{OR}_{v}$ : Average vehicle occupancy or occupation rate by type of vehicle $v$
$D_{i j m v}: \quad$ Total delay of intersection $i$ at the transition step $j$ for movement $m$ by type of vehicle $v(\mathrm{~s})$

$g_{i j m}: \quad$ Effective green time of intersection $i$ at the transition step $j$ for movement or phase $m(s)$

$C_{i j}: \quad$ Cycle length intersection $i$ at the transition step $j(s)$

$\mathrm{OC}_{v}$ : Operating costs by type of vehicle $v$ (\$US/gal)

$\mathrm{FC}_{i j m v}$ : Fuel consumption of intersection $i$ at the transition step $j$ for movement by type of vehicle $v$ (gal)

$\mathrm{ECG}_{e}$ : Environmental costs of the gas emission $e$ (\$US/mg)

$\mathrm{GE}_{\text {ijmve }}$ : Gas emission $e$ in the intersection $i$ at the transition step $j$ for movement $m$ by type of vehicle $v$

$q_{i j m v}$ : Actual or projected demand flow rate of intersection $i$ at the transition step $j$ for movement $m$ by type of vehicle $v(\mathrm{veh} / \mathrm{h})$

Tf: $\quad$ Duration of analysis period (h)

$C_{j}^{c}$ : Common cycle length, at the transition step $j(\mathrm{~s})$

$(\Delta C)_{j}^{c}$ : Change the common cycle length at the transition step $j$ (s)

$C_{i o}$ : Cycle length of intersection $i$ at the step $o$ (before transition period) (s)

$C_{i f}: \quad$ Cycle length of intersection $i$ at the step $n+1=f$ (after transition period) (s)

$(\Delta \varphi)_{i j}$ : Change the offset of intersection $i$ at the transition step $j(\mathrm{~s})$

$\varphi_{i j}: \quad$ Offset of intersection $i$ at the transition step $j$ (s)

$\varphi_{i o}$ : Offset of intersection $i$ at the step o (before transition period) (s)

$\varphi_{i f}: \quad$ Offset of intersection $i$ at the step $n+1=f$ (after transition period) (s)

$(\Delta g)_{i j m}$ : Change the effective green time (split) of intersection $i$ at the transition step $j$ for movement $m$ (s)

$g_{\text {iom }}: \quad$ Effective green time of intersection $i$ at the step $o$ (before transition period) for movement $m$ (s)

$g_{i f m}: \quad$ Effective green time of intersection $i$ at the step $n+1=f$ (after transition period) for movement $m$ (s)

$y_{i j m}: \quad$ Yellow time of intersection $i$ at the transition step $j$ for movement $m$ (s)

$r_{i j m}: \quad$ All red time of intersection $i$ at the transition step $j$ for movement $m(\mathrm{~s})$.

\section{Disclosure}

An earlier version of this article was presented as poster in Transportation Research Board Conference 2015.

\section{Conflicts of Interest}

The authors declare that they have no conflicts of interest. 


\section{Acknowledgments}

The authors would like to acknowledge the Administrative Department of Science, Technology and Innovation (COLCIENCIAS) for having partially financed this work through Contract FP44842-105-2015 (Cod. 1215-669-46846).

\section{References}

[1] D. Schrank, B. Eisele, and T. Lomax, TTI's 2012 Urban Mobility Report, Texas A \& M Transportation Institute. The Texas A \& M University System, 2012.

[2] U.S. Deparment of Transportation, Transportation's role in reducing U.S. greenhouse gas emissions, 2010.

[3] EPA, "Inventory of U.S. Greenhouse gas emissions and sinks: 1990-2010; Notice of availability and request for comments," Federal Register, vol. 77, 2012.

[4] S. M. Chin, O. Franzese, D. L. Greene, H. L. Hwang, and R. C. Gibson, Temporary Losses of Highway Capacity and Impacts on Performance: Phase 2, Department of Energy, USA, 2004.

[5] B. Han, "A new comprehensive sheared delay formula for traffic signal optimisation," Transportation Research Part A: Policy and Practice, vol. 30, no. 2, pp. 155-171, 1996.

[6] J. Kwak, B. Park, and J. Lee, "Evaluating the impacts of urban corridor traffic signal optimization on vehicle emissions and fuel consumption," Transportation Planning and Technology, vol. 35, no. 2, pp. 145-160, 2012.

[7] X. Li, G. Li, S.-S. Pang, X. Yang, and J. Tian, "Signal timing of intersections using integrated optimization of traffic quality, emissions and fuel consumption: a note," Transportation Research Part D: Transport and Environment, vol. 9, no. 5, pp. 401-407, 2004.

[8] D. Gettman, S. G. Shelby, L. Head, D. M. Bullock, and N. Soyke, "Data-driven algorithms for real-time adaptive tuning of offsets in coordinated traffic signal systems," Transportation Research Record, no. 2035, pp. 1-9, 2007.

[9] D. Cohen, L. Head, and S. G. Shelby, "Performance analysis of coordinated traffic signals during transition," Transportation Research Record, no. 2035, pp. 19-31, 2007.

[10] L. Zhang and Y. Lou, "Coordination of semi-actuated signals on arterials," Journal of Advanced Transportation, vol. 49, no. 2, pp. 228-246, 2015.

[11] Y. Bie, Z. Liu, and L. Lu, "Optimization of coordinated signal settings for hook-turn intersections," Journal of Advanced Transportation, vol. 50, no. 2, pp. 197-213, 2016.

[12] W. R. McShane and R. P. Roess, Traffic Engineering, PrenticeHall, Englewood Cliffs, N.J, 2011.

[13] M. C. Coelho, T. L. Farias, and N. M. Rouphail, "A numerical tool for estimating pollutant emissions and vehicles performance in traffic interruptions on urban corridors," International Journal of Sustainable Transportation, vol. 3, no. 4, pp. 246-262, 2009.

[14] Z.-C. Li and X.-Y. Ge, "Traffic signal timing problems with environmental and equity considerations," Journal of Advanced Transportation, vol. 48, no. 8, pp. 1066-1086, 2014.

[15] R. Penabaena-Niebles, V. Cantillo, S. Cuentas, and J. L. Moura, "Transition between signal timing plans: a methodological and analytical overview," Transport Reviews, vol. 34, no. 3, pp. 356$378,2014$.
[16] M. Zheng, H. Xu, K. Zhang, and R. Yao, "Shortest-Way: An Improved Empirical Transition Method for Signal Coordination," Journal of Advanced Transportation, vol. 2017, pp. 1-12, 2017.

[17] E. Lieberman and D. Wicks, "A Rapid Signal Transition Algorithm," Journal of the Transportation Research Board, vol. 509, pp. 158-168, 1974.

[18] M. Abbas, D. Bullock, L. Head, and Trb, "Real-time offset transitioning algorithm for coordinating traffic signals," Advanced Traffic Management Systems and Vehicle-Highway Automation 2001: Highway Operations, Capacity, and Traffic Control, pp. 2639, 2001.

[19] M. F. Selekwa, R. N. Mussa, and A. Chiteshe, "Application of LQ modelling and optimization in urban traffic control," Optimal Control Applications and Methods, vol. 24, no. 6, pp. 331-345, 2003.

[20] J. Lee and B. M. Williams, "Development and evaluation of a constrained optimization model for traffic signal plan transition," Transportation Research Part C: Emerging Technologies, vol. 20, no. 1, pp. 185-198, 2012.

[21] B. Park, P. Santra, I. Yun, and D.-H. Lee, "Optimization of Time-of-Day Breakpoints for Better Traffic Signal Control," Transportation Research Record, no. 1867, pp. 217-223, 2004.

[22] J. Lee, J. Kim, and B. B. Park, "A genetic algorithm-based procedure for determining optimal time-of-day break points for coordinated actuated traffic signal systems," KSCE Journal of Civil Engineering, vol. 15, no. 1, pp. 197-203, 2011.

[23] H. Liu, Travel time prediction for urban networks, Netherlands TRAIL Research School, 2008.

[24] P. Koonce, L. Rodegerdts, K. Lee, S. Quayle, S. Beaird, and C. Braud, Traffic Signal Timing Manual, 2008.

[25] R. Peñabaena-Niebles, V. Cantillo, and J. L. Moura, "Impact of transition between signal timing plans in social cost based in delay, fuel consumption and air emissions," Transportation Research Part D: Transport and Environment, vol. 41, pp. 445456, 2015.

[26] R. Akçelik and M. Besley, "Operating cost, fuel consumption, and emission models in aaSIDRA and aaMOTION," in Proceedings of the in 25th Conference of Australian Institutes of Transport Research (CAITR 2003), pp. 3-5, University of South Australia, Adelaide, Australia, 2003.

[27] B. National Research Council, Transportation Research, HCM 2010 : highway capacity manual, 2010, http://www.hcm2010.org/.

[28] J. Lee, Development and testing of a constrained optimization model for traffic signal plan transition [PhD thesis], Civil Engineering, North Caroline State University, NCSU Institutional Repository, 2009.

[29] R. Akçelik, "Progress in fuel consumption modelling for urban traffic management," 1983.

[30] H. C. Frey, N. M. Rouphail, A. Unal, and J. D. Colyar, "Emissions reduction through better traffic management: An empirical evaluation based upon on-road measurements," 2001.

[31] S. S. Rao and S. Rao, Engineering optimization: theory and practice, John Wiley \& Sons, 2009.

[32] M. Dorigo, M. Birattari, and T. Stützle, "Ant colony optimization," IEEE Computational Intelligence Magazine, vol. 1, no. 4, pp. 28-39, 2006.

[33] M. Dorigo and G. D. Caro, "Ant colony optimization: a new meta-heuristic," in New Ideas in Optimization, C. David, D. Marco, G. Fred, D. Dipankar, M. Pablo, and P. Riccardo, Eds., pp. 11-32, McGraw-Hill Ltd., UK, 1999. 
[34] M. Yaghini, A. Foroughi, and B. Nadjari, "Solving railroad blocking problem using ant colony optimization algorithm," Applied Mathematical Modelling: Simulation and Computation for Engineering and Environmental Systems, vol. 35, no. 12, pp. 5579-5591, 2011.

[35] Y.-C. Chang, V. C. Li, and C.-J. Chiang, "An ant colony optimization heuristic for an integrated production and distribution scheduling problem," Engineering Optimization, vol. 46, no. 4, pp. 503-520, 2014.

[36] B. S. Kim and C. M. Joo, "Ant colony optimisation with random selection for block transportation scheduling with heterogeneous transporters in a shipyard," International Journal of Production Research, vol. 50, no. 24, pp. 7229-7241, 2012.

[37] B. J. Vitins and K. W. Axhausen, "Optimization of large transport networks using the ant colony heuristic," ComputerAided Civil and Infrastructure Engineering, vol. 24, no. 1, pp. 114, 2008.

[38] L. Santos, J. Coutinho-Rodrigues, and J. R. Current, "An improved ant colony optimization based algorithm for the capacitated arc routing problem," Transportation Research Part B: Methodological, vol. 44, no. 2, pp. 246-266, 2010.

[39] J. He and Z. Hou, "Ant colony algorithm for traffic signal timing optimization," Advances in Engineering Software, vol. 43, no. 1, pp. 14-18, 2012.

[40] R. Putha, L. Quadrifoglio, and E. Zechman, "Comparing ant colony optimization and genetic algorithm approaches for solving traffic signal coordination under oversaturation conditions," Computer-Aided Civil and Infrastructure Engineering, vol. 27, no. 1, pp. 14-28, 2012.

[41] L. D’Acierno, M. Gallo, and B. Montella, "An ant colony optimisation algorithm for solving the asymmetric traffic assignment problem," European Journal of Operational Research, vol. 217, no. 2, pp. 459-469, 2012.

[42] R. Peñabaena-Niebles, J. L. Moura Berodia, and V. Cantillo Maza, Diseño y optimización de un modelo matemático para la transición entre el cambio de planes de los tiempos de reparto de los semáforos, 2015, http://www.tesisenred.net/bitstream/handle/ 10803/334972/TesisRPN.pdf? sequence=1.

[43] S. Fankhauser, "Social costs of greenhouse gas emissions: An expected value approach," Energy, vol. 15, no. 2, pp. 158-184, 1994. 


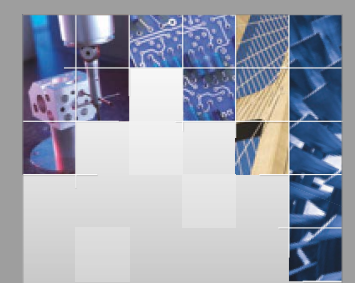

\section{Enfincering}
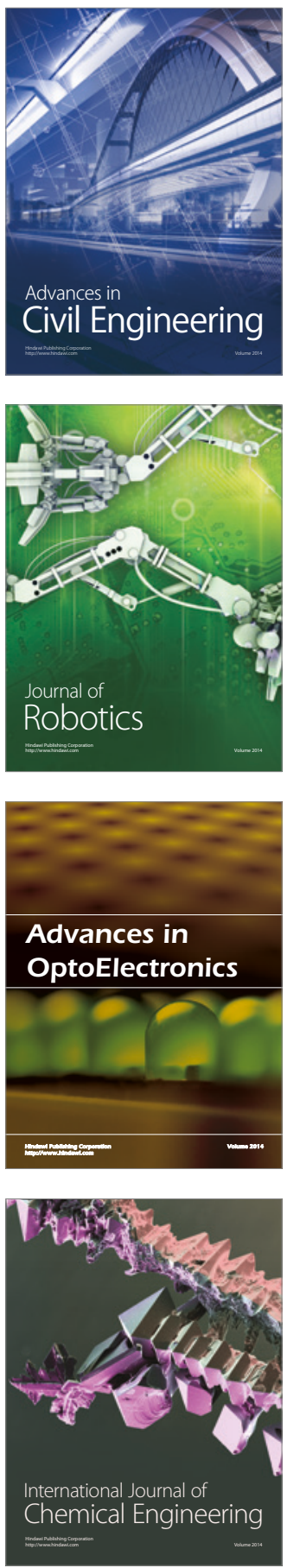



The Scientific World Journal



\section{Hindawi}

Submit your manuscripts at

https://www.hindawi.com
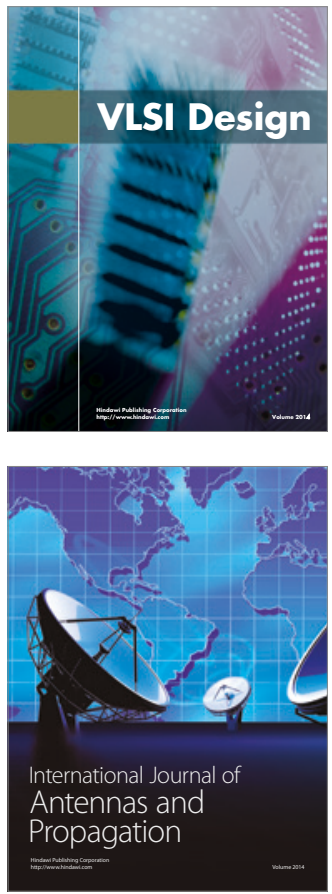

\section{Rotating}

Machinery
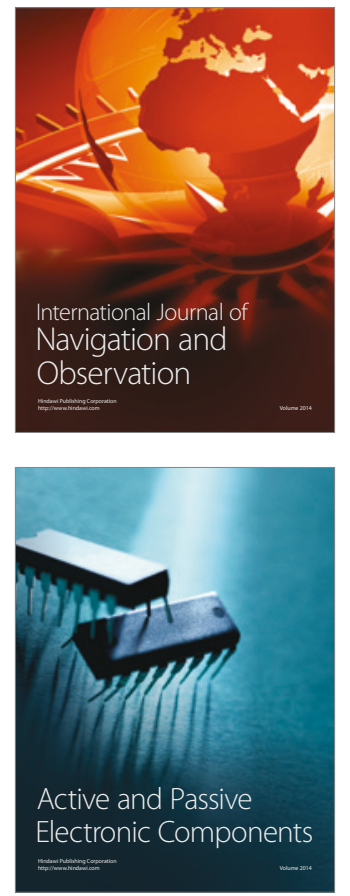
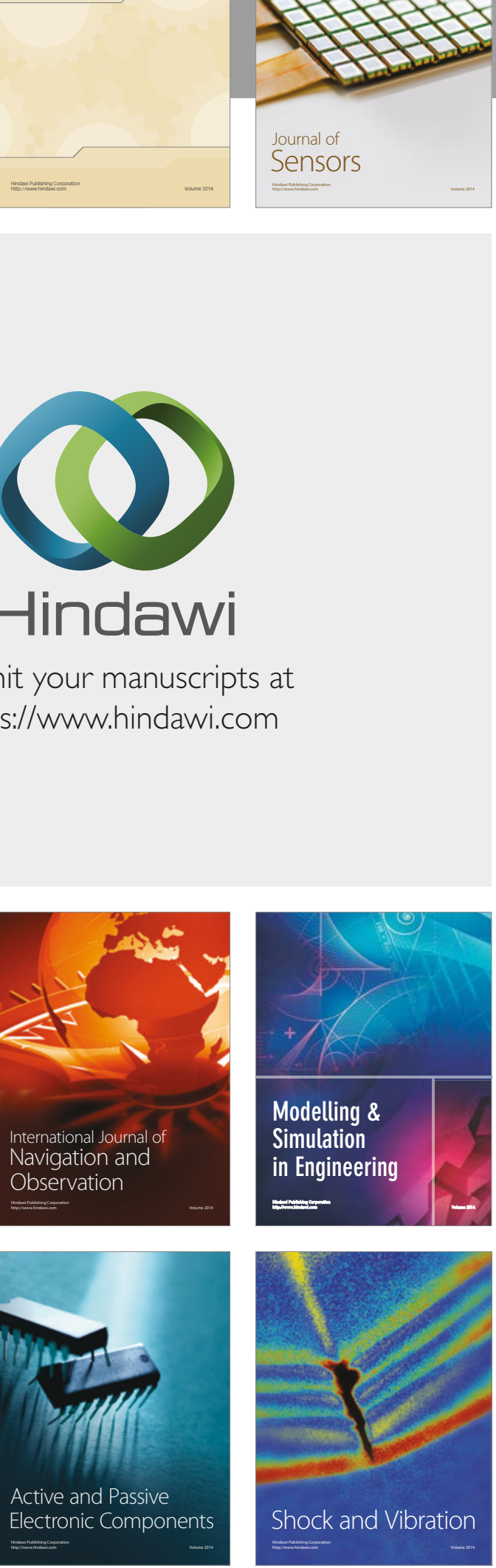
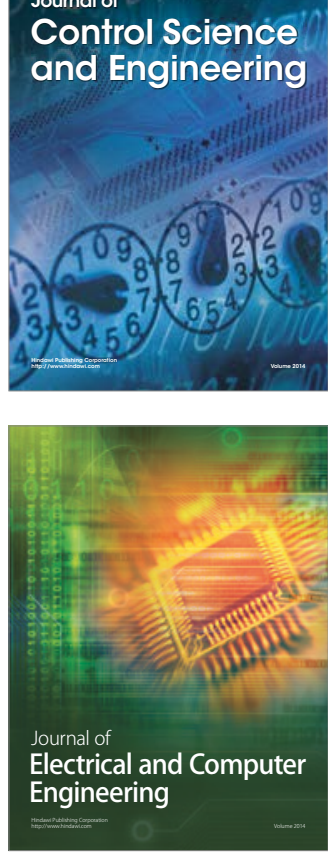

Distributed

Journal of

Control Science

and Engineering
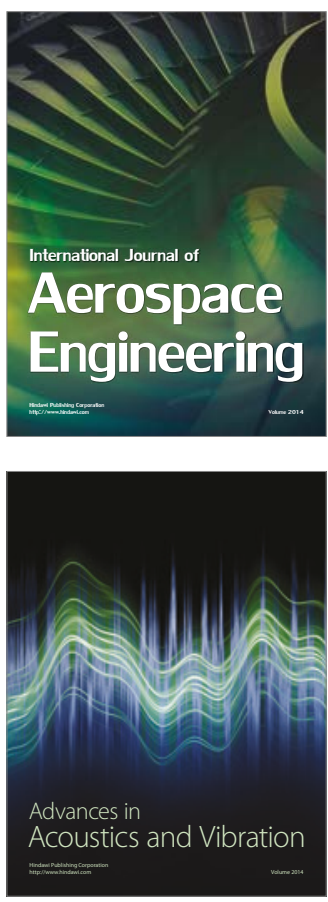

Sensor Networks 